\title{
Papanicolaou durante la menstruación: evaluación de muestras en cuatro hospitales de Lima
}

Papanicolaou sampling during menstruation: evaluation in four Lima hospitals

\author{
Lucy López ${ }^{1}$, José Jerónimo ${ }^{2}$, Rosa Quispe ${ }^{3}$, Rina Takahashi ${ }^{4}$ \\ Subgerente de Sanidad, Municipio Metropolitano de Lima, Perú. \\ ${ }^{2}$ Director del Proyecto VPH PATH, Seattle, Washington, EE UU. \\ ${ }^{3}$ Médico, Departamento de Patología, Hospital de Apoyo María Auxiliadora, Lima, Perú. \\ ${ }^{4}$ Médico Patólogo
}

\begin{abstract}
Resumen
Objetivos: Analizar la factibilidad de obtener una muestra adecuada para el citodiagnóstico durante la menstruación, utilizando la técnica de tomar la muestra cuando el cuello del útero luzca limpio, luego de retirar el flujo menstrual de su superficie. Diseño: Estudio prospectivo, de correlación, de medidas repetidas. Participantes: Mujeres en edad reproductiva. Métodos: Participaron 389 mujeres que acudieron a cuatro hospitales de la región Lima Sur, de quienes se colectó 208 pares de muestras de Papanicolaou: una muestra correspondió al periodo menstrual y otra sin menstruación. La muestra durante la menstruación se obtuvo luego de limpiar suavemente la superficie del cuello uterino con un algodón seco y de esperar a que no hubiera pulso de flujo menstrual a través del os exocervical. Las muestras fueron analizadas separadamente por dos anatomopatólogas. Principales medidas de resultados: Adecuación de las muestras para Papanicolaou tomadas durante la menstruación. Resultados: En dos hospitales en donde las muestras fueron tomadas por personal sensibilizado y entrenado en la técnica, se halló que las muestras con y sin menstruación eran adecuadas para estudio citológico, sin diferencia estadísticamente significativa entre ellas $(p<0,005)$. En los otros dos hospitales, en donde las muestras fueron tomadas mayormente por personal sin entrenamiento, se halló que $75 \%$ de las muestras tomadas durante la menstruación correspondía a extendido hemorrágico, no adecuadas para el estudio del Papanicolaou, habiendo diferencia estadisticamente significativa entre muestras adecuadas con menstruación y sin menstruación $(p<0,005)$. Conclusiones: Los hallazgos nos permiten concluir para este estudio que es posible tomar una muestra adecuada para análisis del Papanicolaou durante la menstruación, si el personal de salud conoce cómo obtener una muestra sin sangre menstrual y está motivado. Este procedimiento puede incrementar el acceso a medidas de prevención del cáncer de cuello uterino.
\end{abstract}

Palabras clave: Cérvix uterino, prueba del Papanicolaou, menstruación.

Abstract

Objectives: To analyze adequate Papanicolaou obtention feasibility during menstruation by thoroughly and softly cleaning the uterine cervix from blood before securing the sample. Design: Correlation prospective study of repeated measurements. Participants: Women in reproductive age. Methods: Three hundred and eighty nine women attending four Lima South region hospitals participated in the study; analysis was done in 208 of them after obtaining a pair of Papanicolaou smears from each woman corresponding to their menstrual and non menstrual periods. The smear taken during menstruation was collected after careful and soft cleaning of the uterine cervix and waiting for absence of menstrual pulse through the cervical os. Two pathologists studied the smears separately. Main outcome measures: Adequacy of Papanicolaou samples obtained during menstruation. Results: Smear samples from menstrual and non menstrual periods were adequate for cytodiagnosis and showed no significant statistical difference $(p<0.005)$ in two hospitals where health providers had been trained and motivated. In the other two hospitals, where most of the samples were collected by health providers without training, $75 \%$ of the smears were obscured by hemorrhagic tissue, inadequate for Papanicolaou cytodiagnosis. There was significant statistical difference between samples obtained with menstruation and without menstruation $(p<0.005)$. Conclusions: It is possible to obtain an adequate sample for Papanicolaou study during menstruation provided health personnel knows the technique to obtain a smear without blood and is motivated to do so. This procedure can increase access to preventive measures for uterine cervix cancer control.

Key words: Uterine cervix, Papanicolaou smear, menstruation.

An Fac med. 2011;72(2):125-30

\section{INTRODUCCIÓN}

El cáncer de cuello uterino es aún una causa importante de enfermedad y muerte en la población de mujeres en el Perú, enfermedad asociada a la prevalencia de infección de virus del papiloma humano (VPH) y a conductas de riesgo ${ }^{(1)}$, pero también a la ineficiencia de programas de prevención, lo cual se agrava por barreras de acceso a los servicios. Entre estas, la práctica frecuente de no tomar la muestra para citodiagnóstico por Papanicolaou durante la menstruación. Si bien actualmente existen nuevos métodos de detección más eficaces, el Papanicolaou está aún vigente y va a tener rol importante como método diagnóstico.

El Perú tiene una población de 5,89 millones de mujeres de 30 y más años, en quienes se concentra el riesgo de desarrollar cáncer de cuello uterino, y una población de 9,51 millones de mujeres 
de 15 y más años ${ }^{(2)}$, cuando se inicia del riesgo de exposición al VPH. De acuerdo a una publicación de la Organización Mundial de la Salud, del 2010, se estima que en el Perú cada año 4446 mujeres son diagnosticadas de cáncer de cuello uterino y 2098 mueren por la enfermedad, alcanzando una tasa bruta de incidencia de 30,9 y mortalidad de 14,6 por 100000 mujeres por año, respectivamente, constituyendo la primera causa de incidencia de cáncer y primera causa de mortalidad en todos los cánceres en la mujer ${ }^{(1)}$.

Para estimar el peso del cáncer de cuello uterino en la salud de la mujer en el Perú, podemos compararlo con la mortalidad materna. Asumiendo que la incidencia y mortalidad del cáncer de cuello uterino atañen a poblaciones de mayor pobreza, acceso limitado a los servicios, la vida en zonas rurales y los niveles bajos de educación ${ }^{(3)}$, estas condiciones son semejantes a las de poblaciones con mayor mortalidad materna. Así, en el año 2009, la cifra absoluta de muerte materna en el Perú fue $482 \operatorname{casos}^{(4)}$, cifra que es la mitad de 12 años atrás y es el resultado de políticas públicas importantes, tales como el seguro materno infantil e integral, la atención con enfoque cultural, el parto vertical y las casas de espera del parto, entre otras, y felizmente sigue descendiendo. Por el contrario, en el caso del cáncer de cuello uterino no ha habido mejora significativa. Más de 2000 mujeres murieron cada año por dicha enfermedad en ese mismo periodo de tiempo y, si siguen las tendencias actuales, se estima que el 2025 tendremos 6882 nuevos casos de cáncer de cuello uterino y 3404 muertes por esta causa ${ }^{(1)}$.

La falta de acceso a servicios para el control de cáncer ha sido relevada en el Plan Nacional de Fortalecimiento para la Prevención y Control del Cáncer en el Perú 2007, que estima en 35500 los casos nuevos de cáncer al año, de los cuales solo 12000 tienen acceso a servicios de salud ${ }^{(5)}$. Es decir, casi dos tercios de la población afectada por cualquier cáncer carecen de servicios. Esto a pesar que, en casos como el del cáncer de cuello uterino, hay gran oportunidad de éxito en el manejo en estadio temprano por procedimientos eficazmente comparables -tales como el cono frío, diatermia, termocoagulación, crioterapia o histerectomía ${ }^{(6)}$ - y el amplio período para la detección y prevención, sabiendo que desde el momento en que se instala una lesión intraepitelial de grado alto, hay 10 años de progreso de la enfermedad hasta que esta lesión se convierta en una lesión invasiva ${ }^{(7)}$.

La prevención por la inmunización contra el VPH es muy prometedora, pero no cubre a toda la población expuesta. En el Perú, se estima que el VPH está presente en 7,5\% de mujeres con citología normal, con presencia de $3,8 \%$ del VPH 16 o 18 en esta población ${ }^{(1)}$. Por todo ello, se ha planteado la importancia de la inmunización antes de la exposición al VPH a través del inicio de relaciones coitales ${ }^{(1)}$. En el Perú, el Ministerio de Salud ha programado la inmunización contra el VPH para las mujeres de 11 años a partir de 2011, estrategia muy pertinente, teniendo en cuenta que $20,9 \%$ de varones y $8,3 \%$ de mujeres entre 15 y 24 años han tenido relaciones coitales antes de la edad de 15 años ${ }^{(1)}$.

Sin embargo, la inmunización es efectiva para el segmento de la población que no se ha expuesto al VPH. Para la población restante, y aún para el seguimiento de la población que recibe inmunización, se requiere combinar estrategias de detección y manejo de la enfermedad. En este contexto, el citodiagnóstico constituye aún un importante procedimiento para la prevención secundaria del cáncer de cuello uterino. El Papanicolaou es utilizado en muchas poblaciones del Perú, solo o en combinación con otros métodos de tamizaje, como la inspección visual con ácido acético (IVAA) ${ }^{(8-11)}$, que fue probada en nuestro país en combinación con el manejo con crioterapia en la región San Martín (12).

Aún con problemas de acceso al servicio y de control de calidad simila- res en las últimas 3 décadas ${ }^{(13,14)}$, en el Perú el Papanicolaou ha sido el procedimiento básico para el control del cáncer de cuello uterino y es realizado en puestos y centros de salud ${ }^{(15)}$. Se estima que 42,9\% de mujeres en el Perú se ha realizado Papanicolaou alguna vez en sus vidas ${ }^{(16)}$, aunque con serias deficiencias. Por ejemplo, en la Diresa Piura, en el 2009, en que se tomó en el año 18000 muestras, se señala una demora de resultados de 1 a 6 meses, lo que resulta en que $30 \%$ a $43 \%$ de las mujeres se pierdan de vista antes de recibir su resultado, hecho especialmente grave para la zona rural ${ }^{(17)}$.

En el tema de prevención secundaria, la primera barrera para el acceso a los servicios de citodiagnóstico es la falta de decisión de la mujer y su gestión para llegar a solicitar el servicio. Una siguiente barrera es el acceso a un servicio efectivo, el mismo que tiene muchos componentes. Uno de ellos, que no ha sido suficientemente discutido, es la limitación impuesta por la práctica de no tomar el Papanicolaou durante los días de menstruación, por el temor de obtener una muestra hemorrágica que no sirve para el citodiagnóstico. Este procedimiento ya está contemplado en las Normas del MINSA ${ }^{(15)}$, que establece que "En caso de presencia de sangrado o flujo, la toma de la muestra se hará previa limpieza cuidadosa del cuello uterino con torunda de algodón seco", y está basado en las condiciones que se debe cumplir para obtener un frotis sin sangre, adecuado para el estudio citopatológico, sin extendido grueso u obscuro y con presencia de células endo y exocervicales ${ }^{(18)}$. Sin embargo, no existe suficiente difusión de las razones por las cuales es posible obtener un Papanicolaou adecuado durante el periodo menstrual y en la práctica persiste la indicación de no tomar el Papanicolaou durante la menstruación. Este temor significa una limitación del servicio en 4/30 días del ciclo menstrual, pero puede ser mayor o incluso excluyente del servicio para esta mujer si vive en la zona rural, en donde el servicio de toma de muestra es más restringido. 
Sin embargo, el riesgo de tomar una muestra hemorrágica durante la menstruación puede ser controlado. En 10 años de práctica de colposcopia en el consultorio de Oncología Ginecológica del Hospital de Apoyo María Auxiliadora de Lima Sur, se observó que el flujo menstrual a través del cuello uterino se manifiesta en finos pulsos, antes que en flujo constante, en relación a la naturaleza del útero compuesto de fibra muscular lisa que mantiene un tono uterino basado en procesos de contracción - relajación ${ }^{(7,19,20,21)}$, lo que permite momentos libres de flujo en los cuales es posible obtener una muestra adecuada.

El presente estudio tiene por objeto analizar la eficacia de la técnica de limpieza de flujo menstrual del cuello uterino para la obtención de una muestra adecuada de Papanicolaou. Y tiene el propósito de incrementar las oportunidades de acceso a los servicios de prevención de cáncer de cuello uterino. La aplicación de la técnica de limpiado del cuello y los resultados del estudio pueden servir también para complementar otros procedimientos utilizados para el manejo del cáncer de cuello uterino.

\section{MÉTODOS}

Se realizó un estudio prospectivo de correlación de medidas repetidas, efectuadas indistintamente antes y durante la menstruación, en mujeres en edad reproductiva concurrentes a centros asistenciales de Lima Sur, específicamente, el consultorio de Oncología Ginecológica del Hospital de Apoyo Departamental María Auxiliadora y los Hospitales Juan Pablo II, Villa María del Triunfo y César López Silva, durante el periodo de un año, entre 1997 y 1998. Los establecimientos fueron seleccionados por tener la mayor demanda de servicios de Papanicolaou entre los establecimientos de la Región de Salud Lima Sur.

La muestra de Papanicolaou durante la menstruación y sin menstruación en las mujeres fue tomada en orden in- distinto, de acuerdo a su aparición en el establecimiento. Participaron 389 mujeres que cumplieron los siguientes criterios: 1) edad entre 20 y 49 años, 2) deseo de participar en el estudio, luego de recibir la explicación de los fines del mismo, y 3) compromiso de retornar para la toma de la segunda muestra. Todas las pacientes proporcionaron consentimiento oral informado antes de ser incluidas en el estudio.

Una vez identificados los establecimientos para el estudio, se invitó a participar a profesionales médicos y obstetrices que reunieran los siguientes criterios: 1) estar a cargo de la toma de muestras de Papanicolaou en sus respectivos hospitales; 2) desear participar en el estudio y completar un taller de capacitación y sensibilización; y, 3) comprometerse a seguir los procedimientos acordados para el estudio.

La capacitación consistió en sensibilización sobre oportunidades perdidas en la detección de cáncer de cuello uterino y entrenamiento en la técnica de limpieza del cuello uterino del flujo menstrual, consistente en los siguientes pasos: 1) colocar espéculo y presentar el cuello uterino; 2) limpiar delicada y cuidadosamente el cuello uterino y paredes de los fondos de saco vaginal con torunda de algodón seco, teniendo cuidado de no causar erosión y sangrado del cuello uterino; 3) esperar un momento en que no hubiera flujo sanguíneo a través del orificio externo del cuello uterino; 4) tomar la muestra tanto del exocérvix como del endocérvix, en un frotis, utilizando espátula de Ayre para exocérvix y escobilla endocervical para el endocérvix ${ }^{(22)}$; y, 5) fijar la muestra en alcohol absoluto de 80 grados por 10 minutos.

En los formatos, se registró nombre y edad de la mujer, además del nombre de la persona responsable de la toma de muestra, sin anotar la condición de menstruación o no. Una copia quedó en el establecimiento y tres copias de los formatos fueron enviados junto con las muestras fijadas al servicio de Oncología Ginecológica del Hospital María
Auxiliadora, en donde funcionó la coordinación del estudio. La relación de los nombres de las participantes en el estudio y la condición de menstruación o no fue registrada separadamente en cada establecimiento.

Para el citodiagnóstico, se contó con la colaboración de dos médicas patólogas que laboraban en el hospital María Auxiliadora y el Instituto Nacional de Enfermedades Neoplásicas, respectivamente. Las profesionales concordaron en: 1) analizar la muestra sin conocer si procedían de periodo con o sin menstruación, y 2) que su opinión iba a ser contrastada con la de otra colega para analizar la reproducibilidad de la opinión técnica ${ }^{(23,24)}$. En cuanto a las muestras, cada uno de los eventos de menstruación o no menstruación era registrado como un evento independiente, de manera que las patólogas recibían las muestras sin conocer si procedían de menstruación o no, dato que solo era conocido por la coordinación del estudio, llevándose de esta manera un control de las dos muestras por cada persona y los resultados de las dos patólogas. Para el reporte del citodiagnóstico, las profesionales emplearon los términos del sistema Bethesda (25,26), analizándose para este estudio solo el primer criterio, es decir, si la muestra era adecuada o inadecuada para hacer el estudio y la razón por la que se la consideraba inadecuada.

Los requisitos para considerar una muestra adecuada para hacer el citodiagnóstico en un frotis fueron los siguientes: 1) contener células epiteliales que provenían del exocérvix y células glandulares que provenían del endocérvix; 2) las características de las células debían permitir determinar si había o no alteración del patrón de desarrollo de ambas estirpes de células; y, 3) la muestra era inadecuada cuando no se podía identificar estas células, porque había escasa celularidad, falta de alguna estirpe celular, era hemorrágica, tenía exceso de células inflamatorias o contenía secreciones mucosas, detritus u otros componentes. 
Los resultados fueron catalogados como: 1) muestra adecuada o adecuada con alguna limitación, por alguna de las siguientes condiciones: muestra inflamatoria, muestra hemorrágica, frotis grueso, escasa celularidad o fijación deficiente; y, 2) muestra inadecuada, por muestra inflamatoria, muestra hemorrágica, frotis grueso, escasa celularidad o mala fijación. Para el estudio, se seleccionó las muestras adecuadas o inadecuadas por muestra hemorrágica.

De las 389 mujeres participantes, se consideró como unidades de análisis 208 participantes, en las cuales se pudo colectar el par de láminas correspondientes, la una con menstruación y la otra sin menstruación. Para el análisis estadístico, se realizó el estudio por separado en cada sede hospitalaria, con el fin de controlar el error de clasificación ${ }^{(27-29)}$, que ocurriría si se analizaba los datos en conjunto sin considerar los factores propios de cada hospital. Por ejemplo, durante el período del estudio hubo rotación del personal de salud entrenado en los hospitales César López Silva y San José.
Se utilizó la prueba chi cuadrado $\left(X^{2}\right)$, de Mc Nemar, método apropiado cuando los eventos no son independientes entre sí ${ }^{(29)}$, como era el caso del presente estudio, en donde la persona participante en el estudio era la misma en ambas muestras. Para el análisis de concordancia entre patólogas, se estimó el estadístico kappa (K).

\section{RESULTADOS}

Participaron 389 mujeres, con edades de 20 a 49 años. Para el análisis se consideró 208 participantes que completaron el par de muestras con menstruación/ sin menstruación, 34 correspondientes al Hospital María Auxiliadora, 21 al Hospital Materno Infantil Juan Pablo II, 128 al Hospital López Silva y 25 al Hospital San José. Los resultados de la comparación de muestras adecuadas o inadecuadas en cada sede son mostrados en la tabla 1 .

En las sedes del Hospital María Auxiliadora y Juan Pablo Segundo, las muestras tomadas sin menstruación y durante la menstruación fueron indistintamente adecuadas para el análisis del Papanicolaou, no hallándose diferencia significativa entre las muestras, con $95 \%$ de probabilidad. En los Hospitales López Silva y San José sí hubo diferencia estadísticamente significativa en los resultados con y sin menstruación. En estas dos últimas sedes, la presencia de extendido hemorrágico fue también significativamente mayor, como se observa en la tabla 2. Estos hallazgos se correlacionaron con la rotación del personal entrenado de los servicios de toma de muestras en los hospitales López Silva y San José.

Para el estudio de reproducibilidad entre patólogas, se utilizó resultados de 395 muestras que habían sido analizadas por las dos patólogas en las cuatro sedes, 183 con menstruación y 194 sin menstruación. Para el caso de las muestras con menstruación, se estimó el estadístico $K=0,44$ y, para las muestras sin menstruación, el $K=0,51$, que corresponden a concordancia moderada entre patólogas.

Tabla 1. Calidad de la muestra por período menstrual y sede hospitalaria.

\begin{tabular}{|c|c|c|c|c|c|}
\hline \multirow{2}{*}{\multicolumn{2}{|c|}{ Hospital María Auxiliadora }} & \multicolumn{2}{|c|}{ Sin menstruación } & \multirow{2}{*}{ Total } & \multirow{2}{*}{$x^{2}$} \\
\hline & & Adecuada & Inadecuada & & \\
\hline \multirow{3}{*}{ Con menstruación } & Adecuada & 28 & 2 & 30 & \multirow{3}{*}{$0,2 \mathrm{NS}$} \\
\hline & Inadecuada & 3 & 1 & 4 & \\
\hline & Total & 31 & 3 & 34 & \\
\hline
\end{tabular}

\begin{tabular}{|c|c|c|c|c|c|}
\hline \multirow{2}{*}{\multicolumn{2}{|c|}{$\begin{array}{l}\text { Hospital Materno Infantil Juan Pablo } \\
\text { Segundo }\end{array}$}} & \multicolumn{2}{|c|}{ Sin menstruación } & \multirow{2}{*}{ Total } & \multirow{2}{*}{$x^{2}$} \\
\hline & & Adecuada & Inadecuada & & \\
\hline \multirow{3}{*}{ Con menstruación } & Adecuada & 13 & 4 & 17 & \multirow{3}{*}{$0,14 \mathrm{NS}$} \\
\hline & Inadecuada & 3 & 1 & 4 & \\
\hline & Total & 16 & 5 & 21 & \\
\hline
\end{tabular}

\begin{tabular}{|c|c|c|c|c|c|}
\hline \multirow{2}{*}{\multicolumn{2}{|c|}{ Hospital Materno Infantil César López Silva }} & \multicolumn{2}{|c|}{ Sin menstruación } & \multirow{2}{*}{ Total } & \multirow{2}{*}{$x^{2}$} \\
\hline & & Adecuada & Inadecuada & & \\
\hline \multirow{3}{*}{ Con menstruación } & Adecuada & 45 & 10 & 55 & \multirow{3}{*}{$\begin{array}{c}37,1 \\
p<0,005\end{array}$} \\
\hline & Inadecuada & 60 & 13 & 73 & \\
\hline & Total & 105 & 23 & 128 & \\
\hline
\end{tabular}

\begin{tabular}{|c|c|c|c|c|c|}
\hline \multirow{2}{*}{\multicolumn{2}{|c|}{ Hospital Materno Infantil San José }} & \multicolumn{2}{|c|}{ Sin menstruación } & \multirow{2}{*}{ Total } & \multirow{2}{*}{$x^{2}$} \\
\hline & & Adecuada & Inadecuada & & \\
\hline \multirow{3}{*}{ Con menstruación } & Adecuada & 8 & 0 & 8 & \multirow{3}{*}{$\begin{array}{c}17 \\
p<0,005\end{array}$} \\
\hline & Inadecuada & 17 & 0 & 17 & \\
\hline & Total & 25 & 0 & 25 & \\
\hline
\end{tabular}


Tabla 2. Proporción de láminas con extendido hemorrágico, según sede y uso de la técnica del estudio.

\begin{tabular}{|c|c|c|c|c|}
\hline & \multirow{2}{*}{ Hospital } & \multirow{2}{*}{ Total de láminas } & \multicolumn{2}{|c|}{ Inadecuadas, con contenido hemorrágico } \\
\hline & & & Número & Porcentaje \\
\hline \multirow{2}{*}{ Aplicaron la técnica del estudio } & María Auxiliadora & 34 & 4 & 12,9 \\
\hline & Juan Pablo II & 21 & 3 & 14,3 \\
\hline \multirow{2}{*}{$\begin{array}{c}\text { No aplicaron o aplicaron parcialmente } \\
\text { la técnica }\end{array}$} & López Silva & 128 & 61 & 47,7 \\
\hline & San José & 25 & 14 & 56 \\
\hline
\end{tabular}

\section{DISCUSIÓN}

El resultado más significativo, de acuerdo al objetivo del estudio, fue la comprobación de que es posible obtener muestra adecuada para el análisis del Papanicolaou durante la menstruación, siendo los resultados comparables con las muestras tomadas sin menstruación. Ello se demostró en los hospitales María Auxiliadora y Juan Pablo II, donde el personal que tomó la muestra estuvo entrenado, constituyendo el entrenamiento un factor importante de la motivación del personal. En contraposición, se ha hallado diferencia significativa en los hospitales López Silva y San José. En estas dos últimas sedes hubo también una proporción alta de muestras con extendido hemorrágico, casi equivalente a 3,5 veces mayor que en María Auxiliadora y Juan Pablo. Debido a cambios en los turnos y rotaciones del personal en los hospitales San José y López Silva, el personal de reemplazo que se hizo cargo de la toma de muestras no había recibido el entrenamiento inicial ni la sensibilización que se brindó al inicio del proyecto, aunque continuó enviando muestras para el estudio.
El error en la clasificación de los grupos en estudio pudo influenciar la magnitud de la asociación entre la exposición y el efecto producido, por analizar los resultados de todos los hospitales juntos en vez de analizarlos por separado, ya que en cada hospital hubo condiciones particulares que influenciaron la calidad de la muestra. Esto se sostiene por el hecho de hallar dos tipos de resultados, dependiendo del entrenamiento y motivación del personal que tomó las muestras. En los hospitales donde no se siguió el procedimiento indicado, hubo 3,5 veces más muestras hemorrágicas que en los hospitales donde permaneció el personal entrenado en la toma de muestra con cuello uterino limpio.

En el presente estudio, la prueba estadística chi cuadrado $\left(\mathrm{X}^{2}\right)$ del tipo de Mc Nemar fue la prueba adecuada que nos permitió analizar las mediciones repetidas en la misma mujer en dos momentos diferentes de su ciclo: cuando menstruaba y cuando estaba sin menstruar. También, se halló que hubo reproducibilidad moderada, pero muy semejante, en las opiniones de las dos patólogas, tanto para las muestras con menstruación como sin menstruación $(K=0,44$ vs, $K=0,51)$. Los resultados de reproducibilidad encontrados son similares a los que se halla en la literatura, que señala que es difícil hallar alta reproducibilidad entre patólogos en la clasificación de los estudios de citología ${ }^{(23)}$, a pesar de los métodos para mejorarla ${ }^{(24)}$.

Como expresamos anteriormente, en el Manual de Normas y Procedimientos para la prevención del Cáncer de Cuello Uterino del Ministerio de Salud del Perú, del año $2000^{(15)}$, se estableció que, en caso de presencia de sangrado o flujo, la toma de la muestra se haría previa limpieza cuidadosa del cuello uterino con torunda de algodón seco. Esto devino del consenso establecido durante la elaboración de la norma, a propuesta de la autora principal del presente estudio, basada en su experiencia de 10 años en la práctica de la colposcopia y manejo clínico para la prevención del cáncer de cuello uterino en el Hospital María Auxiliadora. Por otro lado, no hemos tenido acceso a información que señale la evidencia de por qué es posible obtener una muestra suficiente

Tabla 3. Concordancia de resultados entre las patólogas, para láminas con menstruación y sin menstruación.

\begin{tabular}{|c|c|c|c|c|c|}
\hline \multirow{2}{*}{\multicolumn{2}{|c|}{ Concordancia en láminas con menstruación }} & \multicolumn{2}{|c|}{ Patóloga 1} & \multirow{2}{*}{ Total } & \multirow{5}{*}{$K=0,44$} \\
\hline & & Adecuada & Inadecuada & & \\
\hline \multirow{3}{*}{ Patóloga 2} & Adecuada & 65 & 18 & 83 & \\
\hline & Inadecuada & 31 & 69 & 100 & \\
\hline & Total & 96 & 87 & 183 & \\
\hline
\end{tabular}

\begin{tabular}{|c|c|c|c|c|c|}
\hline \multirow{2}{*}{\multicolumn{2}{|c|}{ Concordancia en láminas sin menstruación }} & \multicolumn{2}{|c|}{ Patóloga 1} & \multirow{2}{*}{ Total } & \multirow{5}{*}{$K=0,51$} \\
\hline & & Adecuada & Inadecuada & & \\
\hline \multirow{3}{*}{ Patóloga 2} & Adecuada & 101 & 16 & 117 & \\
\hline & Inadecuada & 28 & 49 & 77 & \\
\hline & Total & 129 & 65 & 194 & \\
\hline
\end{tabular}


para hacer el estudio del Papanicolaou durante la menstruación, además de la evidencia empírica.

Se concluye que es posible obtener muestras adecuadas para el examen de citodiagnóstico del cuello uterino durante el período menstrual, sin que haya diferencia estadísticamente significativa con la muestra tomada cuando la mujer no está menstruando. La concordancia de opinión consistente de las dos patólogas en el estudio de láminas con y sin menstruación refuerza la conclusión de que una muestra de Papanicolaou puede ser adecuada si se la toma durante la menstruación. Las condiciones para que se cumpla lo anterior dependen del desempeño del personal para tomar una muestra sin extendido hemorrágico. El personal debe tener conocimiento y ser competente en la técnica y estar motivado para ser eficaz y hacer un uso eficiente de los recursos. La aplicación de esta técnica con el fin de disminuir las barreras de acceso al Papanicolaou es viable. Se requiere un mínimo entrenamiento adicional y sensibilización para el personal; el algodón e instrumental necesarios deberán estar disponibles en cualquier establecimiento que tome Papanicolaou. La toma de muestra de Papanicolaou durante la regla significa un incremento de oportunidades para la detección y prevención del cáncer de cuello uterino.

\section{REFERENCIAS BIBLIOGRÁFICAS}

1. World Health Organization/ Institut Català d'Oncologia. Human Papanicolaouilloma virus and related cancers summary report update, Peru. Disponible en www.who.int/hpvcentre). Obtenido el 15 de agosto de 2010.

2. Instituto Nacional de Estadistica e Informática. Censos Nacionales 2007. XI de Población y VI de Vivienda. Lima, Perú, 2007.

3. Organización Panamericana de la Salud. Análisis de la situación del cáncer de cuello uterino en América Latina y el Caribe. 2004.
4. Ministerio de Salud. Reporte de la Dirección General de Salud de las Personas, 2010.

5. Coalición Multisectorial Perú contra el Cáncer. Plan Nacional de Fortalecimiento para la Prevención y Control del Cáncer en el Perú. 2007.

6. Monaghan J. Gynaecology and Obstetrics. En: Dudley H, Carter D, Russell R (editors). Operative Surgery. $4^{\text {th }}$ ed. London: Butterworths; 1987:36-45.

7. MacKay T. Gynecology. En: Tierney LM, McPhee SJ, Papadakis MA (editors). Current Medical Diagnosis \& Treatment, $36^{\text {th }}$ ed. Stamford, CT: /Appleton \& Lange, 1997:671, 675.

8. Blumenthal PD, Gaffikin L, Chirenje ZM, McGrath J, Womack S, Shah K. Adjunctive testing for cervical cancer in low resource settings with visual inspection, HPV, and the Papanicolaou smear. Int J Gynecaecol Obstet. 2001;72(1):47-53.

9. Shlay JC, Dunn T, Byers T, Baron AE, Douglas JM Jr. Prediction of cervical intraepithelial neoplasia grade 2-3 using risk assessment and Human Papanicolaou Virus testing in women with atypia on Papanicolaou smears. Obstet Gynecol. 2000;96(3):410-6.

10. Wu S, Meng L. A comparison of four screening methods for cervical neoplasia. Int J Gynecol Obstet. 2005;91:189-93.

11. Eversole G, Moriarty A, Schwartz M, Clayton A, Souers R, Fatheree L, et al. Practices of participants in the College of American Pathologists: interlaboratory comparison program in cervicovaginal cytology. Arch Pathol Lab Med. 2010;134:331-5.

12. Ministerio de Salud. Proyecto de Prevención de Cáncer de Cuello Uterino en la Región de Salud de San Martín. Reporte. Lima, 2002.

13. Reporte del Congreso Anual de Ex-Residentes del Instituto Nacional de Enfermedades Neoplásicas. Lima, 1992.

14. López L, Ríos Z, Tiburcio H. Prevalencia de citología anormal en Iquitos. Congreso de Cancerologia, Lima. 1984.

15. Ministerio de Salud. Manual de Normas y Procedimientos para la Prevención del Cáncer de Cuello Uterino, Lima, 2000.

16. Lewis MJ. A situational analysis of cervical cancer in Latin America and the Caribbean. En: Health Situation and Trend Analysis, Chronic Noncommunicable Diseases Program and Family Health. PAHO, Washington D.C. 2004.

17. Dirección Regional de Salud de Piura. Reporte de Situación de Cáncer en la Región, 2010.

18. Davey DD, Cox T, Austin M, Birdsong G, Colgan $T$, Howell L, et al. Cervical cytology specimen adequacy: patient management guidelines and optimizing specimen collection. J Low Genital Tract Dis. 2008;12(2):71-81.

19. Bodelsson G, Stjernquist M. Smooth muscle dilation in the human uterine artery induced by substance $P$, vasoactive intestinal polypeptide, calcitonin generated peptide an atrial natriuretic peptide: Relation to endothelium-derived relaxing substances. Human Reprod. 1992;7(9):1185-8.

20. Kimura T, Yoshida Y, Toda N. Mechanisms of relaxation induced by prostaglandins in isolated canine uterine arteries. Am J Obstet Gynecol. 1992;167(5):1409-16.

21. Azuma H, Obayashi S, Hamasaki H, Koyama T, Aso T. Role of endothelium in the human uterine arteries during normal menstrual cycle. $\mathrm{Br} J$ Pharm.1995;114:902-8.

22. Redondo AM, Guerra A, Pinedo G, Garcia R. [Prevention of cervical cancer: Comparison of the sample quality obtained using cotton swab or cervical brush]. Atención Primaria. 2000;26(1):38-41.

23. Ciatto S, Cariaggi MP, Minuti AP, Confortini M, Palli D, Pas L, et al. Interlaboratory reproducibility in reporting inadequate cervical smears- A multicentre multinational study. Cytopathology. 1996;7(6):386-90.

24. Montanari G, Confortini M, Bellomi A, Cocchi V, Dalla Palma P, D'Ambrosio E, et al. Assessment of specimen adequacy reproducibility: An Italian experience. Diagn Cytopathology. 2002;28(4):224-6.

25. Smith AE, Sherman ME, Scott DR, Tabbara SO, Dworkin L, Olson J, et al. Review of the Bethesda System atlas does not improve reproducibility or accuracy in the classification of atypical squamous cells of undetermined significance smears. Cancer. 2000;90(4):201-6.

26. Spires SE, Banks ER, Weeks JA, Banks HW, Davey DD. Assessment of cervicovaginal smear adequacy. The Bethesda System guidelines and reproducibility. Am J Clin Pathology. 1994;102(3):354-9.

27. Gordis L. Epidemiology. $2^{\text {nd }}$ ed. Philadelphia: WB Saunders Co. 2000:246-9.

28. Fisher LD. Biostatistics, a Methodology for the Health Sciences, New York: Wiley, 1993:807.

29. Rothman KJ, Greenland S Modern Epidemiology, Philadelphia: Lippincot - Raven. 1998:254-60;286-7.

\section{Financiamiento:}

El Hospital María Auxiliadora financió el procesamiento de las láminas. El tiempo de los investigadores fue ad honórem. Los costos administrativos fueron asumidos por la investigadora principal

\section{Conflicto de intereses:}

Los investigadores declaran no tener conflicto de intereses en el presente estudio.

Trabajo recibido el 20 de noviembre de 2010 y aceptado para publicación el 14 de mayo de 2011

\section{Correspondencia:}

Dra. Lucy López

lucyherlopez@hotmail.com 